\title{
La relevancia del proyecto estratégico como determinante de la calidad en las universidades
}

\author{
The relevance of the strategic project as a determining factor \\ in the quality of universities
}

\author{
Liliana Pedraja-Rejas ${ }^{1 *}$,Emilio Rodríguez-Ponce ${ }^{2}$
}

\section{RESUMEN}

En el contexto actual, la necesidad de contar con instituciones que lleven a cabo su misión de forma efectiva, resulta fundamental. Sin embargo, ello no es suficiente para desarrollar las labores académicas con calidad. El presente artículo releva a la dirección estratégica como disciplina atingente para generar proyectos estratégicos institucionales de calidad, en el campo de la educación superior. Para este efecto se lleva a cabo una revisión crítica del estado del arte, así como también el análisis de un conjunto de planes generados en universidades iberoamericanas A partir de la revisión teórica y las experiencias observadas se concluye que las instituciones suelen enumerar un conjunto de acciones, las que adolecen de una mirada estratégica y de coherencia con el necesario análisis interno y externo previo. Finalmente, se propone que un buen diseño del proyecto estratégico institucional puede llegar a ser un aspecto diferenciador de las instituciones de educación superior.

Palabras clave: proyecto estratégico, calidad, universidades, estrategia.

\begin{abstract}
In the current context the need for institutions to carry out its mission effectively is essential. However, it is not enough to develop quality academic work. This article replaces the strategic management as the discipline called to generate quality institutional strategic projects in the field of Higher Education. To this end a critical review is carried out, as well as the content analysis of a set of strategic projects generated in Latin American universities. From the theoretical point of view and the experiences observed, it is concluded that institutions tend to list a set of actions, which lack strategic perspective and coherence with the necessary internal and external prior analysis. Finally it is proposed that a good strategic design of the institutional strategic project could become a distinctive and competitive advantage for Higher Education institutions.
\end{abstract}

Key words: strategic project, quality, universities, strategy.

La calidad como constructo posee múltiples acepciones. Ahora bien, desde la perspectiva de la consistencia interna y externa, la calidad se evalúa en función del grado de alineamiento o cumplimiento de la promesa corporativa, que se desprende del proyecto estratégico institucional, en comparación con la realidad lograda por la entidad y por otras organizaciones equivalentes (Pedraja-Rejas y Rodríguez-Ponce 2013).

De este modo, la calidad del diseño del proyecto estratégico institucional debería ser un elemento central que habría de impactar respecto de todas las fases sucesivas del aseguramiento y la gestión de calidad (Pedraja-Rejas, 2014).

No obstante, los estudios internacionales no definen con precisión cómo se configura el proyecto estratégico institucional en las universidades. En efecto, si bien en la literatura existe convergencia en términos de que un proyecto estratégico institucional debe considerar al menos la misión de la universidad, sus propósitos, y sus objetivos (Eaton, 2012, Van Damme et al., 2004), no es menos cierto que salvo escasas excepciones (por ejemplo, Shattock, 2009, 2003; Machado, Farhangmehr y Taylor, 2004) no

\footnotetext{
CEUTA, Universidad de Tarapacá, Arica, Chile.

2 Gobierno Regional de Arica y Parinacota, Arica, Chile.

* Autor para correspondencia: lpedraja@uta.cl
} 
existe una descripción o una pormenorización de los elementos básicos a considerar en el diseño de un proyecto estratégico institucional.

Parece una paradoja, pues lo cierto es que en el acervo de conocimientos se ha logrado consenso con relación a que la medición de la calidad comienza con la observación de la consistencia interna, es decir, comparando la realidad con la promesa institucional; sin embargo, no existe una posición generalmente aceptada acerca de cómo se configura tal promesa. En otros términos, no hay metodologías, ni formatos o modelos predefinidos para diseñar el proyecto estratégico institucional.

Algunos investigadores (por ejemplo, Sha et al. 2011; Machado y Taylor, 2010; Shattock, 2009) han propuesto considerar, derechamente, el diseño estratégico (denominado también dirección estratégica o plan estratégico) como el proyecto estratégico institucional que ha de servir como base para evaluar la calidad de las universidades.

Ciertamente dicha propuesta tiene la ventaja de incorporar los puntos de consenso en la literatura, ya que la misión, los propósitos institucionales y los objetivos estratégicos forman parte del diseño estratégico. No obstante lo anterior, el planteamiento deja por delante la tarea de dimensionar de qué modo los otros aspectos del diseño estratégico como el análisis del entorno, el análisis de recursos y capacidades, el diseño de la estrategia corporativa, competitiva y funcional pueden impactar en la configuración del proyecto estratégico institucional.

La dirección estratégica es el proceso en donde una organización procura alcanzar una ventaja competitiva sustentable que le permita tener éxito. Este proceso implica lograr un alineamiento entre los recursos y las capacidades de la institución con los requerimientos del entorno y del sector específico donde compite. Por lo tanto, la dirección estratégica parece ser lo suficientemente exhaustiva y pertinente para modelar el proyecto institucional de las universidades.

Ahora bien, el proceso de dirección estratégica posee un conjunto de fases, cuyos nombres y énfasis pueden variar en la literatura. No obstante, revisando algunas de las principales publicaciones de difusión sobre el tema es posible identificar las siguientes etapas, comúnmente aceptadas, en un proceso de dirección estratégica (Pedraja-Rejas, 2014):

- Misión, representa la identidad y la personalidad de la organización en el momento actual y de cara al futuro. La misión es la esencia de una institución, su razón de ser, de cara al largo plazo.

- Análisis del entorno general, tiene en cuenta las influencias ambientales, las que a su vez se clasifican por sus fuentes en factores económicos, tecnológicos, demográficos, sociales y gubernamentales.

- Análisis del sector competitivo, considera como elementos básicos de análisis la rivalidad de las organizaciones existentes en el sector, la amenaza de entrada al sector, la amenaza de servicios sustitutos, el poder negociador de los compradores y el poder negociador de los suministradores .

- Análisis de recursos y capacidades, este análisis tiene por objetivo identificar el potencial de la organización para establecer ventajas competitivas mediante la identificación y valoración estratégica de los recursos y capacidades que posee, adicionando aquellos a los que puede acceder. Los recursos pueden ser tangibles o intangibles. Los recursos se traducen en capacidades y competencias para ejecutar actividades concretas en la organización. Las competencias esenciales son aquellas que realizan una aportación relevante al valor final del usuario o a la eficiencia organizativa, y proporcionan una base significativa para ingresar a nuevos sectores.

- Diseño de la estrategia corporativa, en esta fase las instituciones deben evaluar su estrategia corporativa y definir en qué medida va a crecer o sostener su nivel de desarrollo. El crecimiento puede conducir a desarrollar nuevos mercados, nuevos servicios, a la integración vertical, a la diversificación e incluso a la internacionalización. Estas decisiones son parte del diseño de la estrategia de una institución.

- Diseño de la estrategia competitiva, realizada en el nivel de las unidades estratégicas, puede conducir a una segmentación, es decir, a destinar los esfuerzos institucionales a un nicho específico de mercado. Pero además las organizaciones deben considerar a nivel de sus unidades estratégicas de qué manera competirán; es decir, si mediante costos más bajos (liderazgo en costos) o mediante la oferta de un servicio específico (diferenciación). (Rodríguez-Ponce, Pedraja-Rejas, 2009).

- Diseño de la estrategia funcional, definida a nivel de las actividades críticas de la institución, 
que constituyen el quehacer cotidiano y que impactan sobre el desarrollo a largo plazo de las organizaciones. En el caso de las universidades se asocia a la formación de pregrado, postgrado e investigación.

La evidencia empírica en instituciones iberoamericanas permite observar que la mayoría de las universidades generan diseños de la estrategia que resultan insatisfactorios, ya que no logran coherencia entre la estrategia propuesta y su análisis externo e interno; por lo demás, el diseño de las estrategias se basa en un conjunto de acciones que no necesariamente, conducen a la generación de una ventaja competitiva sustentable. Más aún, existen varios planes de desarrollo que destacan por la generación de cientos de acciones denominadas como estratégicas, que son verdaderamente intrascendentes para el logro de los objetivos estratégicos y el cumplimiento de la misión institucional (Rodríguez-Ponce, Pedraja-Rejas, 2013).

En consecuencia, el diseño del proyecto estratégico institucional no es una tarea trivial que sea, por ende, de fácil realización. Por el contrario, el diseño y posterior implementación de un proyecto estratégico institucional es un imperativo que puede diferenciar a unas entidades universitarias de otras $\mathrm{y}$, subsecuentemente, impactar sobre los niveles de calidad que presenten las universidades.

\section{Agradecimientos}

Los autores agradecen a CONICYT por su patrocinio mediante el proyecto FONDECYT 1140026.

\section{Literatura Citada}

Eaton, J.S.

2012. What Future for Accreditation: The Challenge and Opportunity of the Accreditation-Federal Government Relationship. In: Inquiry, Evidence and Excellence. LaCellePeterson, M.; Rigden, D. . Washington: Teacher Education Accreditation Council, pp. 77-88.

Eaton, $\mathrm{J}$.

2010. The Role of Accreditation of Higher Education Institutions. In: International Encyclopedia of Education (Third Edition). Peterson, P.; Baker, E.; McGaw, B. (Eds.), Elsevier, pp. 384-389.

Machado, M.D.L. \& Taylor, J.S.

2010. The struggle for strategic planning in European higher education: the case of Portugal. Research in higher education journal, 6: 1-20.

Machado, M.D.L.; Farhangmehr, M. \& Taylor, J.S.

2004. The Status of Strategic Planning in Portuguese Higher Education Institutions: Trappings or Substance? Higher Education Policy, 17 (4): 383-404.

Pedraja, L.

2014. Proyecto estratégico institucional y la calidad en las universidades: un estudio desde Chile. Pontificia Universidad Católica de Chile. Tesis doctoral no publicada.

Pedraja-Rejas, L.; Rodríguez-Ponce, E.

2013. El desafío de la calidad en las universidades. Idesia, 31 (4): 3-11.
Rodríguez-Ponce, E.; Pedraja-Rejas, L.

2013. Strategic management and quality of universities: an exploratory study from Chile. Interciencia, 38 (1): 35-41.

Rodríguez-Ponce, E.

2009. Las universidades en la sociedad del conocimiento. En: Desafíos y perspectivas de la dirección estratégica de las instituciones universitarias. Arata Andreani, A.; Rodríguez Ponce, E. (Eds). Santiago: Ediciones CNA Chile, 35-68.

Shah, M.; Nair, S.; Wilson, M.

2011. Quality assurance in Australian higher education: historical and future development. Asia Pacific Education Review, 12 (3): 475-483.

Shattock, M.

2009. Dirección estratégica en universidades. En: Desafíos y perspectivas de la dirección estratégica en instituciones universitarias. Arata, A.; Rodríguez-Ponce, E. (eds.). Santiago: CNA-Chile, pp. 117-145.

Van Damme, D.

2004. Standards and Indicators in Institutional and Programme Accreditation in Higher Education: A Conceptual Framework and a Proposal. (127-159). En: Indicators for institutional and programme accreditation in higher/tertiary education.Vlãsceanu, L., \& Barrows, L. (Ed.). Bucarest: UNESCO. 
